\title{
Expression of p53 in leukoplakia and squamous cell carcinoma of the oral mucosa: correlation with expression of $\mathrm{Ki} 67$
}

\author{
S Kannan, G Jagadeesh Chandran, K Raveendran Pillai, Babu Mathew, K Sujathan, \\ K R Nalinakumary, $M$ Krishnan Nair
}

\begin{abstract}
Aim-To study p53 expression in relation to proliferative status in normal and nondysplastic, dysplastic and malignant lesions of the oral mucosa.

Method-The standard avidin-biotin complex (ABC) immunohistochemical staining method was used to study the expression of p53 and $\mathrm{Ki67}$ on frozen sections of oral leukoplakias and carcinomas.
\end{abstract}

Results-Of the leukoplakia and carcinoma samples, $70 \%$ expressed p53 in over $5 \%$ of cells. In normal mucosa less than $5 \%$ of cells expressed p53. The proliferation index, as assessed by expression of $\mathrm{Ki67}$, was highest in the malignant lesions $(43 \%)$ and lowest in normal mucosa (11\%). Statistical analysis revealed that expression of both p53 and Ki67 was correlated significantly with the histopathological stage of the tumour. However, expression of p53 was not correlated with that of Ki67. In leukoplakia lesions with proliferative features p53 immunostaining was less intense than in non-proliferative lesions; this difference was statistically significant. Conclusions-These results emphasise the potential of Ki67 and p53 as biomarkers of carcinogenesis in oral cancer and may also serve as intermediate points for cancer prevention programmes, such as the oral chemopreventive trials. Factors other than p53 may have a more important role in the deregulation of proliferation in pre-malignant oral lesions. (F Clin Pathol: Mol Pathol 1996;49:M170-M175)

Keywords: p53, Ki67, oral leukoplakia, oral squamous cell carcinoma, tumour progression, immunohistochemistry.

Division of Community Oncology Babu Mathew

Division of Dental Surgery

K R Nalinakumary

Division of Radiation Oncology

M Krishnan Nair

Correspondence to: Dr S Kannan, Division of Cancer Research, Regional Cancer Centre,

Thiruvananthapuram 695011 , India.

In recent years more emphasis has been placed on cancer prevention programmes and related studies. In these programmes, success has relied on the development of efficient, early detection methods to identify patients at high risk of developing cancer. Several clinical and aetiological factors have been identified as markers for the detection of these high risk groups for various malignancies. ${ }^{1}$ However, these factors are not efficient and studies are currently underway to identify biological markers for this purpose. ${ }^{2}$ In this regard, unravelling of biological processes during the preneoplastic stages is of paramount importance. ${ }^{3}$
Of the pre-malignant lesions, oral leukoplakia has greatest relevance in the study of the biology of carcinogenesis because the oral cavity is easily accessible for clinical examination ${ }^{4}$ and also because it has a multistage carcinogenesis pathway and of the concept of field cancerisation. ${ }^{5}$ The oral carcinogenesis pathway can be broadly classified histopathologically into normal, non-dysplastic, dysplastic, carcinoma-insitu, and invasive carcinoma. ${ }^{4}$ Molecular biological studies on oral cancer have identified several genetic aberrations in the cells during various tumour stages. ${ }^{6} 7$

Mutations in the p53 tumour suppresser gene are the most common genetic changes in humans cancers and are regarded as early events in carcinogenesis. ${ }^{8-11}$ In pre-malignant and malignant oral lesions, alterations in p53 expression have been reported both at protein and DNA levels. ${ }^{12-18}$ As cancer is characterised by uncontrolled cell proliferation, markers of proliferation, such as Ki67 and proliferating cell nuclear antigen, have been studied extensively in neoplastic lesions. ${ }^{19} \mathrm{Ki} 67$ is a nuclear antigen which is present in the perichromosomal region during mitosis and seems to be a non-histone protein, representing a new class of cell cycle maintaining proteins. ${ }^{20}$ Studies on proliferation markers in lesions of the oral mucosa have shown that expression of $\mathrm{Ki} 67$ is correlated with the severity of the lesion. ${ }^{21-24}$ Studies on the relation between p53 and the proliferation index in various malignancies, including lesions of the oral mucosa, have been reported previously. ${ }^{25-30}$ Our aim was to elucidate the relation, if any, between expression of p53 and Ki67 in normal, non-dysplastic, dysplastic, and neoplastic lesions of the oral mucosa and to determine whether these markers have potential as early indicators of malignancy.

\section{Methods}

Fifty tissue samples, five of normal oral mucosa, 30 of leukoplakia and 15 of invasive carcinoma, were studied. Demographic details were recorded for all patients and included age, sex and whether they used tobacco products. Only those patients with oral leukoplakia and carcinoma at non-keratinising sites of the oral cavity were included. A $4 \mathrm{~mm}$ punch biopsy specimen was collected from each patient, immediately snap frozen and stored in liquid nitrogen. Cryostat sections, $5 \mu \mathrm{m}$ thick, were cut from each biopsy specimen and fixed in cold acetone. One section from each biopsy 
Table 1 Ki67 and p53 nuclear positivity in normal, non-dysplastic, dysplastic, and neoplastic lesions of the oral mucosa

\begin{tabular}{|c|c|c|c|c|c|c|c|c|}
\hline \multirow[t]{3}{*}{ Histopathology } & \multicolumn{8}{|c|}{ Percentage nuclear positivity } \\
\hline & \multicolumn{4}{|c|}{ Ki67 } & \multicolumn{4}{|l|}{$p 53$} \\
\hline & $0-5$ & $6-25$ & $26-60$ & $61-99$ & $0-5$ & $6-25$ & $26-60$ & $61-99$ \\
\hline Normal $(n=5)$ & 3 & 2 & - & - & 5 & - & - & - \\
\hline Non-dysplastic $(n=23)$ & 7 & 6 & 9 & 1 & 5 & 8 & 7 & 3 \\
\hline Dysplastic $(n=7)$ & 1 & 2 & 4 & - & 1 & 5 & 1 & - \\
\hline \multicolumn{9}{|l|}{ Squmaous cell carcinoma } \\
\hline Well differentiated $(n=9)$ & - & 3 & 5 & 1 & 3 & 1 & 5 & - \\
\hline Moderately differentiated $(n=4)$ & - & 2 & - & 2 & 1 & - & 1 & 2 \\
\hline Poorly differentiated $(n=2)$ & - & - & 2 & - & - & - & 1 & 1 \\
\hline
\end{tabular}

specimen was stained with haematoxylin and eosin for histopathological examination. Dysplasia in leukoplakia samples and differentiation in carcinoma specimens were graded as recommended by the World Health Organisation (WHO). ${ }^{31}{ }^{32}$

The standard avidin-biotin complex (ABC) (Unitect immunohistochemistry detection system, Oncogene Science Inc, USA) immunohistochemical staining method was used to detect expression of p53 and $\mathrm{Ki} 67$, as described previously, ${ }^{19}$ using primary monoclonal antibodies directed against p53 [Pab1801 (Oncogene Science, USA) and DO7 (Dako, Glostrup, Denmark)] and Ki67 (Ki67; Dako). Pab1801 and Ki67 were used at a dilution of 1 in 10 and DO7 at a dilution of 1 in 20 . Negative controls (lacking primary antibody) were also included in each assay to assess the specificity of staining. Aminoethylcarbazole (AEC) was used as the chromogen and the slides were counterstained with Mayer's haematoxylin.

The intensity of immunohistochemical staining was graded as follows: negative, mild, moderate, or intense. The pattern of expression was also analysed semiquantitatively by counting the number of positive cells per 100 basal/basaloid cells and was recorded as a percentage. The area with the maximum number of positive cells was considered in each section. The slides were coded and examined blindly.

The relation between the pattern of expression of both antigens and tumour progression was evaluated statistically. The grade of staining was converted into numerical scores as follows: negative $=0 ;$ mild $=2 ;$ moderate $=4 ;$ and intense $=6$. The percentage of positive cells was also scored: $0=0-5 \% ; 2=6-25 \% ; 4=$ $26-60 \% ; 6=61-99 \%$. Tumour progression was classified as follows: normal nonkeratinising mucosa, stage 0 ; non-dysplastic mucosa, stage 1 ; dysplastic mucosa, stage 2 ; all invasive cancer lesions were grouped together and classified as stage 3 . As the scores were not continuous variables, statistical analysis was done using non-parametric methods. The pattern of expression of each protein was compared between histopathological groups using the Mann-Whitney U and Wilcoxon rank sum W tests. Kruskal-Wallis one way analysis of variance was used to test the significance of alterations in expression of p53 and Ki67 during the various stages of tumour progression. The association between the staining pattern and stage of tumour progression was assessed by Spearman's rank correlation analysis.
Results with confidence intervals above $95 \%$ $(\mathrm{p}<0.05)$ were considered significant.

\section{Results}

HISTOPATHOLOGY

Seven $(23 \%)$ of the 30 patients with leukoplakia had low grade dysplastic features. Excluding dysplastic features, the leukoplakias were classified into proliferative (hyperplastic) and non-proliferative (hyperkeratosis) subtypes based on the presence of basal cell hyperplasia, proliferation and elongation of rete pegs, and thickening of the entire epithelium. Thus, the proliferative group comprised seven leukoplakias, three of which exhibited dysplastic features. Of the carcinomas, nine were well differentiated, four were moderately differentiated and remaining two were poorly differentiated. Comparative analysis of the patients' clincial details and histopathological features of the lesions did not reveal any significant relations.

\section{IMMUNOHISTOCHEMISTRY}

Table 1 shows the pattern of expression of Ki67 and p53 in the various oral lesions studied. Both antigens were immunolocalised to the nucleus of basal and parabasal cells of the normal and leukoplakic epithelium (figs 1A and 1B). The two p53 antibodies produced almost identical staining patterns in all tissues evaluated. Interestingly, Ki67 positivity was more pronounced in parabasal than in basal cells. In carcinomas immature basaloid cell nuclei were positive for both antigens (figs 1C and 1D). The staining intensity of both antigens was correlated with the percentage of positive cells-that is, staining was more intense in lesions with larger numbers of positive cells and less so in lesions with fewer positive cells.

In normal mucosa less than $5 \%$ of cells expressed p53 in all cases, while in two cases more than $20 \%$ of cells expressed Ki67. For convenience, $25 \%$ immunopositivity was taken as the cut off value to differentiate low from high antigen expression. $\mathrm{Ki} 67$ and p 53 were highly expressed in $10(45 \%)$ of the 23 non-dysplastic lesions. Ki67 was highly expressed in four $(58 \%)$ of the seven dysplastic lesions, whereas p53 was highly expressed in one (13\%) only (figs $1 \mathrm{~A}$ and $1 \mathrm{~B})$. Ten $(67 \%)$ of 15 carcinomas were strongly positive for both Ki67 and p53 (figs $2 \mathrm{~A}$ and $2 \mathrm{~B}$ ). With regard to the grade of differentiation in carcinomas, poorly differentiated lesions showed more 
Table 2 Univariate analysis to test the significance of differences in the percentage positivity of Ki67 and 553 between different histopathological groups by Mann-Whitney $U$ and the Wilcoxon Rank Sum $W$ tests ( $Z$ value; $p$ value)

\begin{tabular}{llll}
\hline Lesion & Normal & Non-dysplastic & Carcinoma \\
\hline $\begin{array}{c}\text { Non-dysplastic } \\
\text { Ki67 }\end{array}$ & $1.7081 ; 0.0876$ & & \\
p53 & $2.8212 ; 0.0048^{\star}$ & & $2.1316 ; 0.0330^{\star}$ \\
$\begin{array}{l}\text { Dysplastic } \\
\text { Ki67 }\end{array}$ & $2.0598 ; 0.0394^{\star}$ & $0.6789 ; 0.4972$ & $0.8250 ; 0.4094$ \\
p53 & $2.7105 ; 0.0067^{\star}$ & $0.8568 ; 0.3916$ & $1.0371 ; 0.2997$ \\
Carcinoma & & \\
Ki67 & $2.9742 ; 0.0029^{\star}$ & & \\
p53 & $2.5814 ; 0.0098^{\star}$ & & \\
\hline
\end{tabular}

ॠSignificant.

Table 3 Bivariate analysis to test the relation between the pattern of expression of both Ki67 and p53 and tumour progression in the oral mucosa

\begin{tabular}{llllll}
\hline \multirow{2}{*}{ Marker } & \multicolumn{2}{l}{ Kruskal-Wallis one way ANOVA } & & \multicolumn{2}{l}{ Spearman's rank correlation } \\
\cline { 2 - 3 } & $\chi^{2}$ & $p$ value & & $r_{s}$ & $p$ value \\
\hline Ki67 & 10.6074 & $0.0140^{\star}$ & & 0.4475 & $0.001^{\star}$ \\
p53 & 10.2394 & $0.0166^{\star}$ & & 0.3020 & $0.033^{\star}$ \\
\hline
\end{tabular}

ॠSignificant. to the proliferative status of the leukoplakia lesions. This relation was not observed in carcinomas.

STATISTICAL ANALYSIS

The mean percentage of Ki67 and p53 positivity in the various histological groups is presented graphically in fig 3. Univariate analysis showed a significant difference between the expression pattern of the normal and other histopathological groups for both Ki67 and p53. No significant differences were observed between non-dysplastic, dysplastic and carcinoma groups (table 2). When the patterns of expression of $\mathrm{p} 53$ and $\mathrm{Ki} 67$ were compared in the different leukoplakias with respect to proliferative status, $\mathrm{p} 53$ showed a significant difference ( $p=0.0169$ ), but for Ki67 the difference was statistically insignificant ( $\mathrm{p}=$ 0.0758 ). Kruskal-Wallis one way analysis of variance and Spearman's rank correlation analyses showed significance in the expression pattern for both the $\mathrm{Ki} 67$ and p53 in relation to tumour progression (table 3). Correlation analysis of the proliferative status of leukoplakias and staining showed a significant negative correlation coefficient for $\mathrm{p} 53 \quad\left(\mathrm{r}_{\mathrm{s}}=\right.$ $-0.4436 ; p=0.014)$, but not for $\mathrm{Ki67}\left(\mathrm{r}_{\mathrm{s}}=\right.$ $0.3297 ; p=0.075)$. Correlation analysis of the percentage of positivity and intensity of staining also showed a significant positive correlation for both the Ki67 $\left(r_{s}=0.5143 ; p=\right.$ $0.0001)$ and $p 53\left(r_{s}=0.7482 ; p=0.0001\right)$. The association between the percentage $\mathrm{Ki} 67$ and p53 positivity was insignificant $\left(r_{s}=\right.$
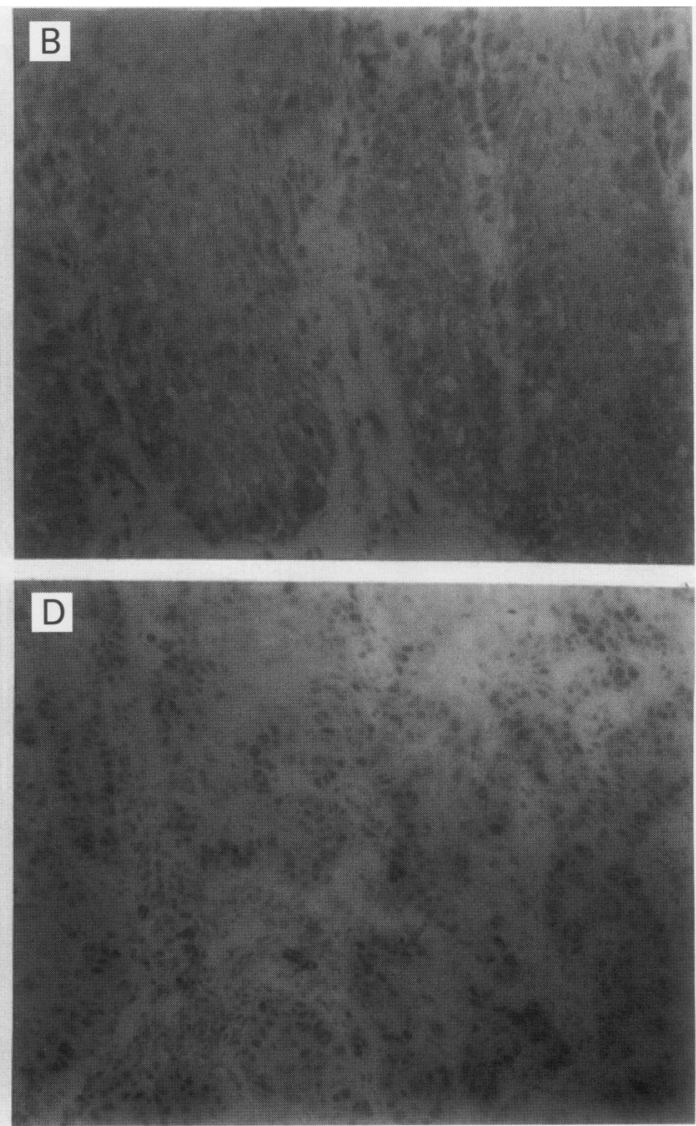

Figure 1 Immunohistochemical pattern of expression of $(A)$ Ki67 and (B) p53 in dysplastic oral leukoplakia and of $(C)$ Ki67 and (D) p53 in well differentiated squamous cell carcinoma of the mouth (original magnification, $\times 100$ ). 

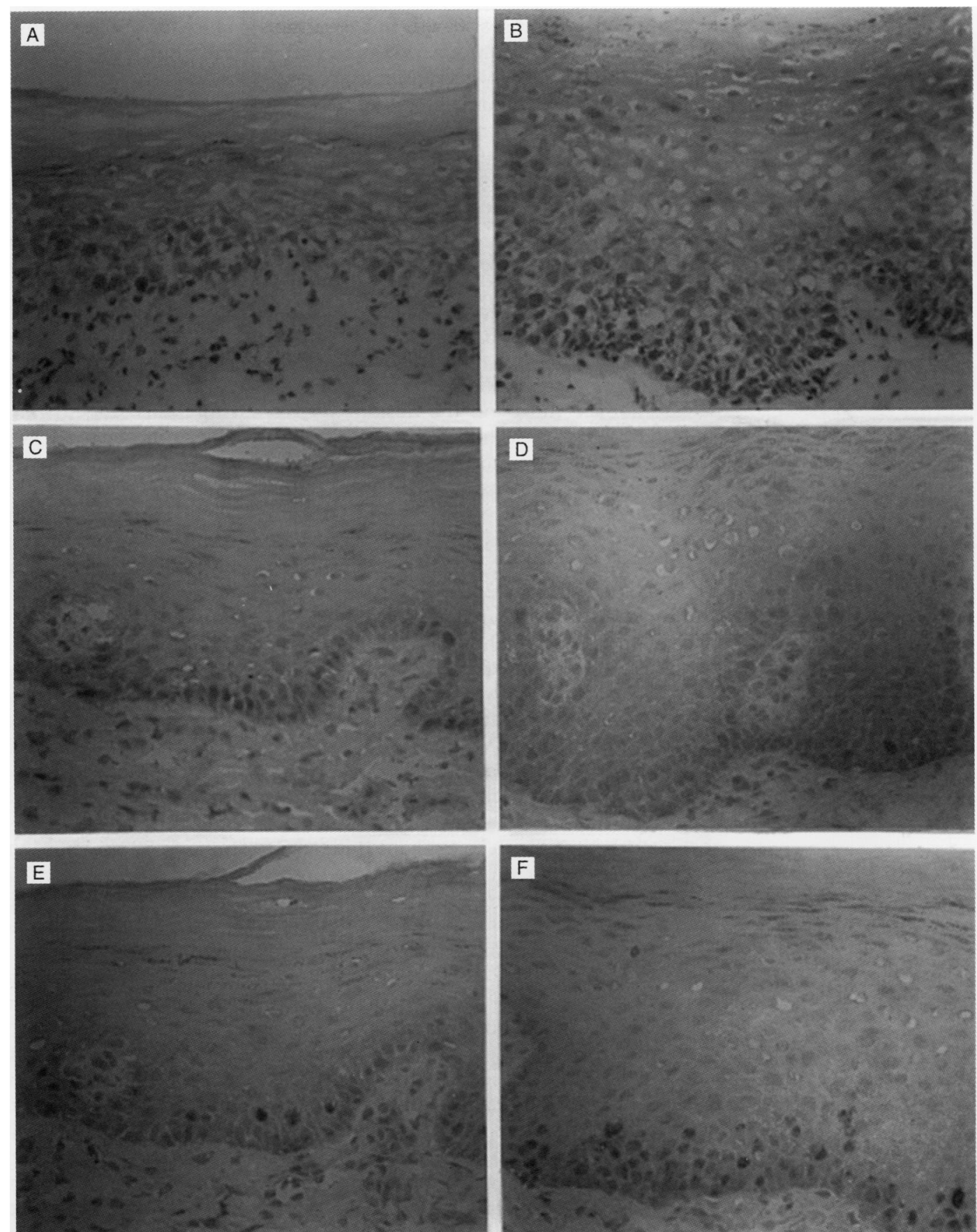

Figure 2 Immunohistochemical pattern of expression of Ki67 and $p 53$ in non-proliferative ( $A$, haematoxylin and eosin; $C$, $p 53 ; E, K i 67)$ and proliferative $(B$, haematoxylin and eosin; $D, p 53 ; F, K i 67)$ oral leukoplakia lesions (original magnification, $\times 250$ ).

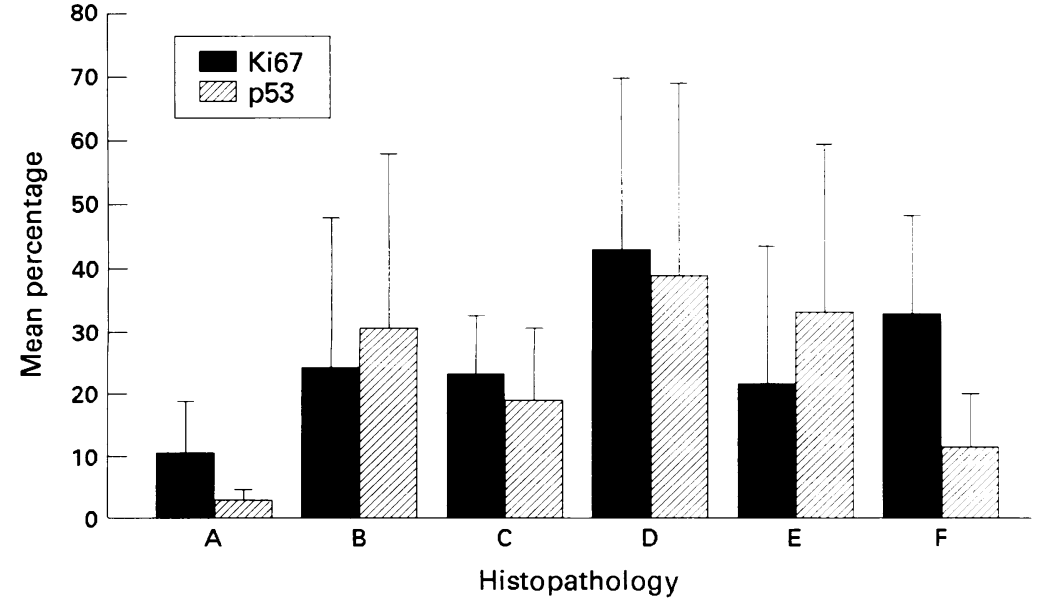

Figure 3 Mean (SD) percentage of Ki67 and p53 nuclear positivity in various oral lesions. $A=$ normal mucosa; $B=$ non-dysplastic leukoplakia; $C=$ dysplastic leukoplakia; $D$ $=$ squamous cell carcinoma $; E=$ non-proliferative leukoplakia $F=$ proliferative leukoplakia.
$0.2636 ; \mathrm{p}=0.064)$, while the correlation coefficient was positive for the whole population. In leukoplakias alone, no significant correlation was observed $\left(r_{\mathrm{s}}=-0.1552 ; \mathrm{p}=0.413\right)$.

\section{Discussion}

Mutations in the p53 gene are the most common genetic changes observed in human carcinomas. ${ }^{8-11}$ Mutant p53 protein is more stable than its wild type counterpart and thus can be visualised by immunohistochemistry. ${ }^{10}{ }^{11}$ Accumulation of $\mathrm{p} 53$ protein in the cell is generally considered to be the result of mutation of the p53 gene. These mutations lead to uncontrolled cell proliferation, resulting in further genetic abnormalities and finally in malignancy. ${ }^{8}$ Therefore, the nature of the p53 gene and the proliferative status of a cell are 
closely linked and the loss of this linkage is one of the main causes of tumour formation and is considered to be an early event in this process. ${ }^{9}$ To analyse the proliferative status of a cell or tissue various markers are available, of which $\mathrm{Ki} 67$ is the most widely used and reliable. It recognises a proliferation related nuclear antigen present at all phases of cell cycle except $\mathrm{G}_{0}{ }^{20}$

In the present study $70 \%$ of the samples showed greater than $5 \%$ positivity for p53 while normal mucosa expressed p53 in less than $5 \%$ of cells. Of the leukoplakias $78 \%$ of the non-dysplastic and $86 \%$ of the dysplastic lesions expressed p53 in more than $5 \%$ of cells. In carcinomas most of the lesions (73\%) exhibited high expression of $\mathrm{p} 53$. The proliferation index, as evaluated by expression of $\mathrm{Ki} 67$, was highest in carcinomas (43\%) and lowest in normal mucosa (11\%). In leukoplakias expression of $\mathrm{Ki} 67$ was intermediate and was almost identical in non-dysplastic (24\%) and dysplastic (23\%) lesions. Previous studies of p53 expression in oral pre-malignant and malignant lesions showed a range of positivity of 35 to $90 \%$ and also reported a correlation between p 53 positivity and the severity of the lesion. ${ }^{14}{ }^{16-18}{ }^{33}$ However, a study from Sri Lanka found a low prevalence $(11 \%)$ of p53 expression in oral carcinoma. ${ }^{34}$ The reason for this notable variation in $\mathrm{p} 53$ positivity from that observed in the present study, as well as in other reports, is difficult to explain. Both studies were carried out on frozen sections using the same antibody. Interestingly, population differences in ras mutation have been reported previously in oral carcinoma for Indian and UK samples. ${ }^{35} 36$

A significant relation between the staining pattern and tumour progression has been observed for both $\mathrm{Ki} 67$ and p53. Earlier studies using various proliferation markers also reported a similar association between the proliferative index and tumour progression in oral mucosa. $^{21-24}$ However, no correlation was found between the pattern of expression of p53 and Ki67 in oral pre-malignant and malignant lesions. This suggests that $\mathrm{p} 53$ expression have a direct effect on the proliferative status of oral lesions, supporting the conclusions of earlier reports. ${ }^{25-30}$ Of the two markers, Ki67 exhibited a more pronounced association with tumour progression, although its expression was more heterogeneous in oral leukoplakias. This variation in expression may be of prognostic importance for the early detection of pre-malignant lesions. These results emphasise the potential use of Ki67 and p53 as biomarkers of carcinogenesis in oral cancer and also may serve as intermediate points for cancer prevention programmes, such as the oral chemopreventive trials. $^{13} \begin{array}{lllll}14 & 18 & 33 & 37\end{array}$

At the histopathological level a higher percentage of $\mathrm{p} 53$ positivity was observed in in non-proliferative hyperkeratotic lesions (mean $33.22 \pm 25.8)$ than in their proliferative counterparts (mean $11.43 \pm 8.58$ ). This observation is not compatible with current thinking on the stability of wild type and mutated p53 protein-that is, immunohistochemical posi- tivity for $\mathrm{p} 53$ protein in a cell is suggestive of a mutation in the p53 gene with the subsequent induction of proliferation by the mutated protein. In the present study fewer cells expressed p53 in the proliferative than in nonproliferative leukoplakia lesions. The proliferative status of the lesions was confirmed by $\mathrm{Ki} 67$ expression; the proliferative lesions have a mean percentage Ki67 positivity of $32.57 \pm$ 21.72 whereas that of the non-proliferative lesions was $21.48 \pm 21.72$. The results of some recent studies have also raised doubts about the concept of the stability of p53 proteins and inferences of immunohistochemical results (reviewed by Soussi et $a l^{10}$ ). Some mutations lead to transcription or translation errors in the p53 gene, which result in the arrest of p53 protein synthesis and negative staining on immunohistochemistry. Tumours with nonsense or frameshift mutations also result in the production of unstable, truncated proteins, which are also negative on immunohistochemistry. These types of mutations are estimated to account for less than $15 \%$ of human tumour mutations, depending on the cancer. $^{10}$ Overexpression without p53 gene mutation has also been observed in breast carcinoma. ${ }^{38}$ The study on the oral cancers from Sri Lanka also found that of the few immunohistochemically p53 positive cases, none had mutations in exons 5-8, which are considered to be a "hot spot" for mutation. ${ }^{39}$ Accumulation of $\mathrm{p} 53$ proteins in normal cells followed by certain stresses, such as ultraviolet irradiation ${ }^{40}$ and exposure to carcinogens, ${ }^{9}$ has also been reported. Hence, it is not possible to determine whether p53 positivity observed in the present study was a result of mutation or the accumulation of normal p53 induced by carcinogens present in tobacco, as all of the patients were chronic betel quid chewers. However, previous studies, including those on head and neck tumours, have compared the results of p53 immunoexpression with those of molecular methods and most of them found a significant association between the two techniques. ${ }^{13} \mathrm{On}$ the basis of this report, the present result can be interpreted as indicating that factors other than p53, or which act prior to the appearance of p53 aberrations, may be responsible for alterations in the proliferation index of these pre-malignant oral lesions. Molecular analysis of the p53 gene in these tissues to confirm the present findings is essential, and is currently underway. Although the relation between the p53 and Ki67 expression is not clear, especially in pre-malignant oral lesions, the significant correlation between tumour progression and expression of these antigens suggests that they may be useful biomarkers of oral carcinogenesis.

This work was supported by a young scientist grant SRßY-B12[9] 1 from the Department of Science and Technology, Gov$12[9] 1$ from the Dep
ernment of India.

\footnotetext{
1 Ames DN, Gold LS, Willet WC. The causes and prevention of cancer. Proc Natl Acad Sci USA 1995;92:5258-65.

2 Sidransky D. Molecular markers in cancer: can we make Sidransky $D$. Molecular markers in cancer: can
better predictions? Int $\mathcal{f}$ Cancer 1995;64:1-2. 
3 Sporn MB. Carcinogenesis and cancer: different perspectives on the same disease. Cancer Res 1991;51:6215-18.

4 Meyskens FL Jr. Biology and intervention of the premalignant processes. Cancer Bull 1991;43:475-80.

5 Boyd NM, Reade PC. Mechanism of carcinogenesis with particular reference to the oral mucosa. 7 Oral Pathol Med 1988;17:193-201.

6 Scully C, Burkhardt A. Tissue markers of potentially malignant human oral epithelial lesions. $f$ Oral Pathol Med 1993;22:246-56.

7 Scully $\mathrm{C}$. Oncogenes, tumor suppressors and viruses in oral squamous carcinoma. $f$ Oral Pathol Med 1993;22:337-47.

8 Furihata $\mathrm{M}$, Sonobe H, OhtsukiY. The aberrant $\mathrm{p} 53$ protein (review). Int $\mathcal{F}$ Oncol 1995;6:1209-26.

9 Levine AJ, Perry ME, Chang A, Silver A, Dittmer D, Wu M, et al. The 1993 Walter Hubert Lecture: the role of the p53 tumor-suppressor gene in tumorigenesis. $\mathrm{Br} \mathcal{F}$ Cancer 1994;69:409-16.

10 Soussi T, Legros Y, Lubin R, Ory K, Schtichtholz B. Multifactorial analysis of $\mathrm{p} 53$ alteration in human cancer factorial analysis of p53 alteration

11 Harris CC, Hollstein M. Clinical implications of the p53 tumor-suppressor gene. $\mathrm{N} \mathrm{Engl} \mathcal{F}$ Med 1993;329:13-27.

12 Field JK. The role of oncogenes and tumor-suppressor genes in the aetiology of oral, head and neck squamous cell carcinoma. $\mathcal{F} R$ Soc Med 1995;88:35P-9P.

13 Ahomadegbe JC, Barrois M, Fogel S, Le Bihan ML, DoucRasy S, Duvillard P, et al. High incidence of p53 alteration (mutation, deletion, overexpression) in head and neck primary tumors and metastases; absence of correlation with clinical outcome. Frequent protein overexpression in normal epithelium and in early non-invasive lesions. Oncogene 1995;10:1217-27.

14 Regezi JA, Zarbo RJ, Regev E, Pisanty S, Silverman S, Gazi D. p53 protein expression in sequential biopsies of oral
dysplasias and in situ carcinomas. F Oral Pathol Med 1995; 24:18-22.

15 Thomas S, Brennan J, Martel G, Frazer R, Montesano R Sidransky $\mathrm{D}$, et al. Mutations in the conserved regions of p53 are infrequent in betel-associated oral cancers from Papua New Guinea. Cancer Res 1994;54:3588-93.

16 Kaur J, Srivastva A, Ralhan R. Overexpression of p53 protein in betel-and tobacco-related human oral dysplasia and squamous-cell carcinoma in India. Int 7 Cancer 1994; 58:340-5.

17 Girod SC, Kramer C, Knufermann R, Krueger GR. p53 expression in the carcinogenesis in the oral mucosa. $f$ Cell Biochem 1994;56:444-8.

18 Warnakulasuriya KA, Johnson N. Expression of mutant nuclear phosphoprotein in oral carcinoma and potentially malignant oral lesions. $\mathcal{F}$ Oral Pathol Med 1992;21:404-8.

19 Begg AC. Critical appraisal of in situ cell kinetic measurements as response pedictors in human tumors. Semin Radiat Oncol 1993;3:144-51.

20 Schluter C, Duchrow M, Wohlenberg C, Becker MH, Key $\mathrm{G}$, Flad HD, et al. The cell proliferation-associated antigen of antibody Ki67: a very large, ubiquitous nuclear protein with numerous repeated elements, representing a new kind of cell cycle-maintaining proteins. $f$ Cell Biol 1993; of cell cycle-

21 Valente G, Orecchia R, Gandolfo S, Arnaudo M, Ragona R Kerim S, et al. Can Ki67 immunostaining predict response to radiotherapy in oral squamous cell carcinoma? $\mathcal{f}$ Clin Pathol 1994;47:109-12.

22 Zoller J, Flentje M, Sinn P, Born IA. Evaluation of AgNOR and Ki67 antigen as cell kinetic parameters in oral dysplasias and carcinomas. Anal Cell Pathol 1994;7:77-84.
23 Shin DM, Voravud N, Ro JY. Sequential increases in proliferating cell nuclear antigen expression in head and neck tumorigenesis: a potential biomarker. $\mathcal{f}$ Natl Cancer Inst 1993;85:971-5.

24 Tsuji T, Sasaki K, Kimura Y,Yamada K, Mori M, Shinozaki F. Measurement of proliferating cell nuclear antigen F. Measurement of proliferating cell nuclear antigen (PCNA) and its clinical application in

25 Nylander K, Nilsson P, Mehle C, Roos G. p53 mutations, protein expression and cell proliferation in squamous cel carcinomas of the head and neck. Br $\mathcal{F}$ Cancer 1995; 71:826-30.

26 Nylander K, Stenling R, Gustafsson H, Zakrisson B, Roos G. p53 expression and cell proliferation in squamous cell carcinomas of the head and neck. Cancer 1995;75:87-93.

27 Slootweg PJ, Koole R, Hordijk GJ. The presence of $p 53$ protein in relation to Ki67 as cellular proliferation marker in head and neck squamous cell carcinoma and adjacent in head and neck squamous cell carcinoma and adjacent dysplastic

28 Warnakulasuriya KA, Johnson NW. Association of overexpression of $\mathrm{p} 53$ oncoprotein with the state of cell proliferation in oral carcinoma. $\mathcal{F}$ Oral Pathol Med 1994;23:246-50.

29 Girod SC, Pape HD, Krueger GR. p53 and PCNA expression in carcinogenesis of the orophary

30 Girod SC, Krueger G, Pape HD. p53 and Ki67 expression in preneoplastic and neoplastic lesions of the oral mucosa. in preneoplastic and neoplastic lesions of

31 Wahi PN, Cohen B, Luthra UK, Torloni H. Histological typing of oral and oropharyngeal tumors. Geneva: World Health Organisation, 1971

32 World Health Organisation Collaborating Centre For Ora Precancerous Lesions. Definition of leukoplakia and related lesions: an aid to studies on oral precancer. Oral Surg 1978;46:518-39.

33 Shin DM, Kim J, Ro JY, Hittelman J, Roth JA, Hong WK, et al. Activation of p53 gene expression in premalignant al. Activation of p53 gene expression in premalignant 1994;54:321-6.

34 Ranasinghe AW, Warnakulasuriya KA, Johnson NW. Low prevalence of expression of p53 oncoprotein in oral carcinomas from Sri Lanka associated with betel and tobacco chewing. Eur $\mathcal{f}$ Cancer B Oral Oncol 1993;29B:147-50.

35 Saranath D, Chang SE, Bhoite LT, Panchal RG, Kerr IB, Mehta AR, et al. High frequency mutation in codons 12 and 61 of $\mathrm{H}$-ras oncogene in chewing tobacco-related human oral carcinoma in India. $\mathrm{Br} F$ Cancer 1991; 63:573-8.

36 Chang SE, Bhatia P, Johnson NW, Morgan PR, McCormick $\mathrm{F}$, Young $\mathrm{B}$, et al. Ras mutations in United Kingdom examples of oral malignancies are infrequent. Int $\mathcal{f}$ Cancer 1991;48:409-12.

37 Shin DM, Hittelman WN, Hong WK. Biomarkers in upper aerodigestive tract tumorigenesis: a review. Cancer Epidemiol Biomarkers Prev 1994;3:697-709.

38 Coles C, Condie A, Chetty U, Steel CM, Evans HJ, Prosser J. p53 mutations in breast cancer. Cancer Res 1992; 52:5291-8

39 Ranasinghe A, MacGeoch C, Dyer S, Spurr N, Johnson NW. Some oral carcinomas from Sri Lankan betel/tobacco
chewers overexpress p 53 oncoprotein but lack mutations chewers overexpress p53 oncoprotein but lack
in exons 5-9. Anticancer Res 1993;13:2065-8.

40 Hall PA, McKeen PH, Menage HD, Dover R, Lane DP. High levels of $\mathrm{p} 53$ protein in UV-irradiated normal human skin. Oncogene 1993;8:203-7. 